\title{
OPEN SETS OF DIFFEOMORPHISMS HAVING TWO ATTRACTORS, EACH WITH AN EVERYWHERE DENSE BASIN
}

\author{
ITTAI KAN
}

\begin{abstract}
We announce the discovery of a diffeomorphism of a three-dimensional manifold with boundary which has two disjoint attractors. Each attractor attracts a set of positive 3-dimensional Lebesgue measure whose points of Lebesgue density are dense in the whole manifold. This situation is stable under small perturbations.
\end{abstract}

\section{INTRODUCTION}

Through the numerical experiments and theoretical works of many researchers we have gained some understanding of a dynamical system which is "typical" in the "standard" setting; that is, the dynamical system $f: M \rightarrow M$ is a smooth map and $M$ is a smooth $n$-dimensional manifold. Special circumstances change the meaning of "typical" and add possibilities to the list of what we are likely to see, say, by requiring $f$ to be in some particular subset of smooth maps on $M$. For example, if $f$ is symplectic, then we see the KAM tori and other phenomena of Hamiltonian dynamics; or if $f$ has symmetries, we are likely to see "symmetry-breaking" bifurcations. The setting we examine here is where $f$ maps some fixed submanifold $K \subset M$ of dimension $k<m$ to itself ( $f$ is invariant on $K$ ). That is, we discuss some new phenomena which are "typical" in the sense that they occur for some large set of perturbations of $f$ which continue to map $K$ to itself. This is the natural setting for modeling physical systems with constraints or reflectional symmetries.

We find some unexpected new limiting behaviors in the new setting. More precisely, the $\omega$-limit set of a point $\mathbf{x} \in M$ is the set $\omega(\mathbf{x})=\bigcap_{k} \overline{U_{l \geq k} f^{l}(\mathbf{x})}$, the forward limit set of $\mathbf{x}$ under iteration by $f$. We say that a compact set $A$ is an attractor if $\{\mathbf{x} \in M: \omega(\mathbf{x})=A\}$ has positive $n$-dimensional Lebesgue measure and the basin of attraction of $A$ is $\mathscr{B}(A)=\{\mathbf{x} \in M: \omega(\mathbf{x}) \subset A\}$.

In the standard setting the basins of attraction typically seem to be open sets, although the boundaries of these basins are often fractal sets [1]. Certainly, in the standard setting, it has long been known that the only structurally stable attractors are the Axiom A attractors, and these have open basins of attraction; see, for example, [2]. In the setting where we have a fixed invariant manifold $K$, we find that attractors may have basins with some radically new features. We find that some maps have several coexisting attractors whose basins are measure-theoretically dense in each other. That is, any open set which intersects one basin in a set of positive measure also intersects each of the other basins in a set of positive measure. We call such basins intermingled.

Received by the editors March 23, 1993.

1991 Mathematics Subject Classification. Primary 54C35, 58F13.

Supported in part by AFOSR grant F49620-92-J-0033. 
In [3] numerical evidence for intermingled basins is presented in the case of a particular quadratic map of the plane. The main result of this paper is that there are maps for which intermingling can be rigorously shown to occur and, in fact, to persist under perturbation.

Theorem 1. For every $k \geq 1+\alpha$, there is an open set of $C^{k}$ diffeomorphisms of the thickened torus with boundary $\left(T^{2} \times I\right)$ for which there are two coexisting attractors whose basins are intermingled and the union of whose basins has full measure.

In fact, with choice of coordinates $T^{2}=S^{1} \times S^{1}=[0,1) \times[0,1)$ and $I=[0,1]$ we find that a small $C^{k}$ neighborhood of the $C^{\infty}$ diffeomorphism

$$
f(x, y, z)=\left(3 x+y, 2 x+y, z+\cos (2 \pi x)\left(\frac{z}{32}\right)(1-z)\right)
$$

satisfies the theorem. That is, for any diffeomorphism in this neighborhood, the two boundary tori, $A=T^{2} \times\{0\}$ and $B=T^{2} \times\{1\}$, are each attractors; the union of their basins has full 3-dimensional Lebesgue measure; and their basins are intermingled.

A result similar to Theorem 1 can be obtained in two dimensions if we abandon the requirement of invertibility. For the annulus we have the following theorem.

Theorem 2. For every $k \geq 1+\alpha$, there is an open set of $C^{k}$ maps of the annulus $\left(S^{1} \times I\right)$ which send the boundary to the boundary for which there are two coexisting attractors whose basins are intermingled and the union of whose basins has full measure.

With choice of coordinates $S^{1}=[0,1)$ and $I=[0,1]$, we find that a small $C^{k}$ neighborhood of the $C^{\infty}$ map

$$
f(x, z)=\left(3 x, z+\cos (2 \pi x)\left(\frac{z}{32}\right)(1-z)\right)
$$

satisfies the theorem. That is, for any map in this neighborhood, the two boundary circles, $A=S^{1} \times\{0\}$ and $B=S^{1} \times\{1\}$, are each attractors; the union of their basins has full 2-dimensional Lebesgue measure; and their basins are intermingled.

Remark 1. Although the basins for these attractors are measure-theoretically large, they are small topologically (they are meager, the countable union of nowhere dense sets). It is easy to show that this is generally true in the case of a continuous map where there are two disjoint attractors whose basins are intermingled.

Remark 2. There are many other definitions of attractor and basin in use today $[4,5]$. The theorems of this paper hold for most of the measure-theoretic definitions.

The proofs of Theorems 1 and 2 are long and technical. In Section 2 we give a proof of Theorem 2 restricted to the case where $f$ is the map given by (2). This proof is elementary and provides insight into the general proofs. In Section 3 we discuss the modifications needed to prove the general case of Theorems 1 and 2 . 


\section{AN EXAMPLE ON THE ANNULUS}

The proof of Theorem 1 is similar to the proof of Theorem 2 but contains additional technicalities which may obscure the main ideas of this paper. In addition, the 2-dimensional dynamics of the map of the annulus given by (2) are much easier to visualize than the the map of the thickened torus given by (1); so in this section we show that for the map of the annulus given by (2), the two boundary circles are each attractors, the union of their basins has full 2-dimensional Lebesgue measure, and their basins are intermingled.

Our coordinates for the annulus will be $(x, z) \in[0,1) \times[0,1]$ with $(0, z)$ identified with $(1, z)$. We note that for $f$, the $x$-coordinate function $f_{x}$ depends only on the $x$-coordinate, and thus $f$ maps vertical line segments to vertical line segments. We use $m$ to denote 2-dimensional Lebesgue measure and $m_{x}$ to denote the measure on curves which is locally 1-dimensional Lebesgue measure of the orthogonal projection to the $x$-axis.

We note that the points $(0,0),(0,1),\left(\frac{1}{2}, 0\right)$, and $\left(\frac{1}{2}, 1\right)$ are each fixed points of $f$. Also, the Jacobian matrix has the lower triangular form

$$
\begin{aligned}
J f(x, z) & =\left(\begin{array}{ll}
{\left[D_{x} f_{x}\right](x, z)} & {\left[D_{z} f_{x}\right](x, z)} \\
{\left[D_{x} f_{z}\right](x, z)} & {\left[D_{z} f_{z}\right](x, z)}
\end{array}\right) \\
& =\left(\begin{array}{cc}
3 & 0 \\
-\frac{\pi z}{16}(1-z) \sin (2 \pi x) & 1+\frac{(1-2 z)}{32} \cos (2 \pi x)
\end{array}\right) .
\end{aligned}
$$

Lyapunov exponents and stable manifolds. We now review some basic facts and definitions from the theory of dynamical systems. In particular, we discuss Lyapunov exponents and stable manifolds.

The Lyapunov exponent for a vector $\vec{v}$ with base point $(x, z)$ is the exponential rate at which the vector length grows under iteration by $f$. That is,

$$
\lambda(\vec{v})=\lim _{n \rightarrow \infty} \frac{1}{n} \ln \left\|J f^{n}(x, z) \vec{v}\right\|
$$

if the limit exists and where $f^{n}$ denotes the $n$-th iterate of $f$. Each point in $\mathbf{x} \in A$ has at most two Lyapunov exponents. One is called the normal Lyapunov exponent and measures the exponential rate of expansion in the direction perpendicular to the boundary circle $A$. By the diagonal form of the Jacobian on $A$ this exponent is

$$
\lambda_{\perp}(\mathbf{x})=\lim _{n \rightarrow \infty} \frac{1}{n} \sum_{k=1}^{n} \ln \left|\left[D_{z} f_{z}\right]\left(f^{k}(\mathbf{x})\right)\right|,
$$

if the limit exists.

In order to organize which points are attracted to which attractor, the following definitions are useful. The stable manifold of a point $\mathbf{x}$ is the set of points which converge to the orbit of $\mathbf{x}$, that is, $W^{\mathrm{s}}(\mathbf{x})=\{\mathbf{y} \in M$ : $\left.\lim _{n \rightarrow \infty}\left\|f^{n}(\mathbf{x})-f^{n}(\mathbf{y})\right\|=0\right\}$. Since $f$ maps vertical segments to vertical segments and the restriction of $f$ to a single vertical segment is invertible, it is easy to see that if the point $(\hat{x}, \rho)$ is in the stable manifold for a point $\mathbf{x}=(x, 0) \in A$, then every point $(\hat{x}, z)$ with $z \leq \rho$ is also in the stable manifold of the point $\mathbf{x}$. Thus the stable manifold of the point $\mathbf{x}$ is a union 
of (possibly trivial) vertical line segments whose bottoms terminate in $A$. The component of $W^{\mathrm{s}}(\mathbf{x})$ which contains $\mathbf{x}$ is called the immediate stable manifold of $\mathbf{x}$ and is denoted by $W^{\text {is }}(\mathbf{x})$. Since $f$ is expanding in the $x$-direction and is 3-to-1, we see that the stable manifold of $\mathbf{x}$ is a union of inverse images of forward images of the immediate stable manifold of $\mathbf{x}$. That is, $W^{\mathrm{s}}(\mathbf{x})=\bigcup_{n \geq 0} f^{-n}\left(f^{n}\left(W^{\text {is }}(\mathbf{x})\right)\right)$.

Lemmas. We now show that $A$ is on the average contracting in the normal direction. This will imply that $A$ is an attractor and that the immediate stable manifold of almost every point in $A$ is a vertical line segment of positive length. The analogous results for the other boundary circle $B$ follow by symmetry.

Lemma 2.1. For $m_{x}$-almost every point $\mathbf{x} \in A$ the normal Lyapunov exponent is negative.

Proof of Lemma 2.1. Since 1-dimensional Lebesgue measure $m_{x}$ on $A$ is ergodic and invariant under $f$, the ergodic theorem tells us that almost every $\mathbf{x}=(x, 0) \in A$ has a normal Lyapunov exponent equal to

$$
\lambda_{\perp}=\int_{0}^{1} \ln \left|\left[D_{z} f_{z}\right](x, 0)\right| d x=\int_{0}^{1} \ln \left|1+\frac{\cos (2 \pi x)}{32}\right| d x .
$$

By symmetry we have

$$
\begin{aligned}
\lambda_{\perp} & =2 \int_{0}^{\frac{1}{4}} \ln \left|1-\frac{\cos (2 \pi x)}{32}\right|+\ln \left|1+\frac{\cos (2 \pi x)}{32}\right| d x \\
& =2 \int_{0}^{\frac{1}{4}} \ln \left|1-\left(\frac{\cos (2 \pi x)}{32}\right)^{2}\right| d x<0 .
\end{aligned}
$$

We now show that for every point in $A$ whose normal Lyapunov exponent is negative the immediate stable manifold has positive length. Since $m_{x}$-almost every point of $A$ has a normal Lyapunov exponent equal to $\lambda_{\perp}<0$ and all immediate stable manifolds are vertical line segments, we then easily see that $m(\mathscr{B}(A))>0$.

Lemma 2.2. If $\hat{\mathbf{x}}=(\hat{x}, 0) \in A$ and $\lambda_{\perp}(\hat{\mathbf{x}})=\lambda_{\perp}<0$, then $W^{\text {is }}(\hat{\mathbf{x}})$ has positive length.

Corollary. The basin of $A$ has positive 2-dimensional Lebesgue measure.

Proof of Lemma 2.2. Assume $\hat{\mathbf{x}}$ is as in the hypothesis of the lemma. Choose a positive $c$ sufficiently small so that for all $x$ we have that $z<c$ implies $|\ln |\left[D_{z} f_{z}\right](x, 0)|-\ln |\left[D_{z} f_{z}\right](x, z)||<\left|\frac{\lambda_{1}}{4}\right|$. Since $\lambda_{\perp}(\hat{\mathbf{x}})<0$, the quantity $\tau(\hat{x})$ defined as follows

$$
\tau(\hat{x})=\sup _{n \geq 0}\left\{\frac{-3 n \lambda_{\perp}}{4}+\sum_{k=0}^{n} \ln \left|\left[D_{z} f_{z}\right] f^{k}(\hat{\mathbf{x}})\right|\right\},
$$

is finite.

By the Mean Value Theorem and the Chain Rule for differentiation, we see that if $f_{z}^{k}(\hat{x}, z)<c$ for all $0 \leq k \leq n$, then

$$
\ln \left|f_{z}^{n+1}(\hat{x}, z)\right|<\ln (z)-\left|\frac{n \lambda_{\perp}}{4}\right|+\sum_{k=0}^{n} \ln \left|\left[D_{z} f_{z}\right] f^{k}(\hat{\mathbf{x}})\right| \leq \ln (z)+\tau(\hat{\mathbf{x}})+\frac{n \lambda_{\perp}}{2} \text {. }
$$


In fact, if $z$ is chosen to be less than $c \exp (-\tau(\hat{x}))$, then $f_{z}^{n+1}(\hat{x}, z)<$ $c \exp \left(\frac{n \lambda_{1}}{2}\right)<c$. Thus by induction we see that the length of $W^{\text {is }}(\hat{\mathbf{x}})$ is at least $c \exp (-\tau(\hat{x}))$.

We now show that the points in $A$ whose immediate stable manifolds are long are measure-theoretically dense in the circle $A$. This will imply that the basin of $A$ is measure-theoretically dense in the annulus and by a symmetric argument the basin of $B$ is also measure-theoretically dense and thus the two basins are intermingled.

Lemma 2.3. Let $R_{\rho}$ denote the set $\left\{\mathbf{x} \in A: \omega(\mathbf{x})=A, \lambda_{\perp}(\mathbf{x})<0,\left|W^{\text {is }}(\mathbf{x})\right| \geq\right.$ $\rho\}$. Then, for any $0<\rho<1$ we have $m_{x}\left(R_{\rho}\right)>0$ and the points of 1dimensional Lebesgue density of $R_{\rho}$ are dense in $A$.

Recall that $\mathbf{x}$ is a point of Lebesgue density for a set $C$ if $\lim _{\varepsilon \rightarrow 0} \frac{m\left(\mathscr{N}_{\epsilon}(\mathbf{x}) \cap C\right)}{m\left(\mathscr{N}_{\epsilon}(\mathbf{x})\right)}=$ 1 , where $\mathscr{N}_{\epsilon}(\mathbf{x})$ denotes an $\epsilon$-neighborhood of $\mathbf{x}$. Lebesgue almost every point of a set of positive Lebesgue measure is a point of Lebesgue density for that set.

Corollary. The basins of $A$ and $B$ are intermingled.

Proof of Lemma 2.3. Let $R$ denote the set $\left\{\mathbf{x} \in A: \omega(\mathbf{x})=A, \lambda_{\perp}(\mathbf{x})<0\right\}$. By Lemma 2.1 this is a set of full 1-dimensional Lebesgue measure. By Lemma 2.2 we can fix an $\varepsilon>0$ so that $m_{x}\left(R_{\varepsilon}\right)>0$.

The immediate stable manifold of the fixed point $\mathbf{p}=\left(\frac{1}{2}, 0\right)$ is the vertical line segment $\frac{1}{2} \times[0,1)$ and is the saddle connection between the hyperbolic saddle fixed point $\mathbf{p}$ and the repelling fixed point $\mathbf{q}=\left(\frac{1}{2}, 1\right)$. Let $\theta(x, z)$ denote the branch of $f^{-1}$ which maps the annulus to the vertical strip $\left[\frac{1}{3}, \frac{2}{3}\right) \times$ $[0,1]$. By the vertical structure of $f$ we see that for each $\mathbf{x} \in R_{\varepsilon}$ the vertical line segment $\theta^{k}\left(W^{\text {is }}(\mathbf{x})\right)$ converges to $W^{\text {is }}(\mathbf{p})=\left\{\frac{1}{2}\right\} \times[0,1)$ as $k \rightarrow \infty$. Let $n_{\delta}(\mathbf{x})$ denote the smallest positive integer $n$ such that $\left|\theta^{n}\left(W^{i s}(\mathbf{x})\right)\right| \geq$ $(1-\delta)$. It is easy to see that for positive $\delta$ the function $n_{\delta}(\mathbf{x})$ is a bounded function on $R_{\varepsilon}$. We denote the supremum of $n_{\delta}(\mathbf{x})$ on $R_{\varepsilon}$ by $n_{\delta}$. Since $\left|\left[D_{z} f_{z}\right](x, z)\right| \leq \frac{33}{32}$ for all $(x, z)$ in the annulus, for any $\theta^{n}\left(W^{\text {is }}(\mathbf{x})\right)$ whose length is at least $(1-\delta)$, each of the $3^{k}$ evenly spaced (in the $x$-coordinate) images of $\theta^{n}\left(W^{\text {is }}(\mathbf{x})\right)$ under $f^{-k}$ is a vertical line segment of length at least $1-\left(\frac{33}{32}\right)^{k} \delta$.

So, for a fixed $0<\rho<1$ we find a dense subset $\hat{R}_{\rho}$ of $R_{\rho}$ with immediate stable manifolds of length greater than $\rho$ by taking preimages of stable manifolds whose lengths are almost 1 . That is, for $\rho=1-\delta$ we have

$$
\hat{R}_{\rho}=\bigcup_{k \geq 0} f^{-k}\left(\theta^{n_{\ell(k)}}\left(R_{\varepsilon}\right)\right),
$$

where $\ell(k)=\delta\left(\frac{32}{33}\right)^{k}$. Since $m_{x}\left(R_{\varepsilon}\right)$ is positive, we see that $m_{x}\left(\theta^{n_{\ell(k)}}\left(R_{\varepsilon}\right)\right)$ is also positive. The $3^{k}$ images under $f^{-k}$ of $R_{\varepsilon}$ also each have positive measure and are evenly spaced on $A$, and thus the set $\hat{R}_{\rho}$ has points of 1-dimensional Lebesgue density which are dense in $A$. Since $\hat{R}_{\rho}$ is a subset of $R_{\rho}$, we see that $R_{\rho}$ is as desired.

In order to show that the union of the basins of $A$ and $B$ has full measure, we need to establish some control on the slope of iterated horizontal line segments. 
Lemma 2.4. If $\gamma$ is a horizontal line segment of the form $\gamma=\left[n 3^{-r}\right.$, $\left.(n+1) 3^{-r}\right) \times\{\rho\}$, then $f^{r}(\gamma)=\gamma_{r}$ is the graph of a function $Z(x)$ whose domain is $[0,1)$ and whose derivative is at most $\frac{\pi}{10}$ in absolute value.

Proof of Lemma 2.4. The maximum value of $\left|\left[D_{z} f_{z}\right](x, z)\right|$ is $\frac{33}{32}$, and the maximum value of $\left|\left[D_{x} f_{z}\right](x, z)\right|$ is $\frac{\pi}{16}$; so the lower triangular form of the derivative matrix of (3) allows us to establish easily that slopes whose absolute value is less than $\frac{\pi}{10}$ continue to be slopes whose absolute value is less than $\frac{\pi}{10}$ after iteration by $f$.

Proof of Theorem 2 restricted to the case of $f$. By the corollary of Lemma 2.3 the basins of attraction of $A$ and $B$ are intermingled. In order to show that the complement of $(\mathscr{B}(A) \cup \mathscr{B}(B))$ has 2-dimensional Lebesgue measure zero, we will show that the complement of $(\mathscr{B}(A) \cup \mathscr{B}(B))$ has no points of horizontal 1-dimensional Lebesgue density.

Consider an arbitrary horizontal line segment $\gamma$ as in Lemma 2.4 and its $r$-th iterate $\gamma_{r}$. We assume that $Z(0) \leq \frac{1}{2}$. By Lemma 2.4 the derivative of the function $Z(x)$ is at most $\frac{\pi}{10}$, and thus the function $Z(x)$ is bounded above by $\beta=\frac{1}{2}+\frac{\pi}{10}$. The immediate stable manifolds of the set $R_{\beta}$ each intersect $\gamma_{r}$ in exactly one point, so $m_{x}\left(\gamma_{r} \cap \mathscr{B}(A)\right) \geq m_{x}\left(R_{\beta}\right)$. The image under $f^{-r}$ in $\gamma$ of $\gamma_{r} \cap \mathscr{B}(A)$ is in the basin of attraction of $A$. Since $f^{r}$ is linear in the $x$ coordinate, $m_{x}\left(R_{\beta}\right)$ is a lower bound on the $m_{x}$-measure of the fraction of $\gamma$ which is in the basin of $A$. If $Z(0)>\frac{1}{2}$, then we carry out a similar argument for $B$ instead of $A$. So the set of points which are in neither the basin of $A$ nor the basin of $B$ cannot have any points of horizontal 1-dimensional Lebesgue density and by Fubini's theorem must therefore be a set whose 2-dimensional measure is zero.

\section{The General CASE}

We discuss the technical issues which must be dealt with to prove Theorem 2 in the general case where $\tilde{f}$ is a small $C^{1+\alpha}$ perturbation of $f$. Full details can be found in [6]. There are four main technical complications.

First, the invariant measures on $A$ and $B$ may be perturbed. Fortunately, the perturbed measures remain absolutely continuous and have continuous densities close to 1 . Second, if $\tilde{f}_{x}$ no longer depends only on the $x$-coordinate, vertical lines may no longer be sent to vertical lines. However, the results of Hirsch, Pugh, and Shub [7] on normal hyperbolicity assure us that a kind of almost vertical structure will be preserved. Third, for a smooth curve $\eta$ transverse to the stable manifolds of $A$, the holonomy map $h_{\eta}$ taking a point $\mathbf{x} \in A$ to $\eta \cap W^{\text {is }}(\mathbf{x})$ is not necessarily even Lipschitz. Fortunately, Pésin theory [8-10] allows us sufficient control of the Radon-Nikodym derivative of the holonomy map. Fourth, distortion estimates are needed when pulling back measures from $\gamma_{r}$ to $\gamma$, since the map $\tilde{f}$ need not be linear in the $x$-coordinate.

\section{ACKNOWLEDGMENT}

We thank Charles Pugh, James C. Alexander, James A. Yorke, John Milnor, Lai-Sang Young, and David Lavine for their helpful discussions. 


\section{REFERENCES}

1. S. W. McDonald, C. Grebogi, E. Ott, and J. A. Yorke, Fractal basin boundaries, Phys. D 17 (1985), 125-153.

2. R. Bowen, On Axiom A diffeomorphisms, CBMS Regional Conf. Ser. in Math., vol. 35, Amer. Math. Soc., Providence, RI, 1978.

3. J. C. Alexander, I. Kan, J. A. Yorke, and Zhiping You, Riddled basins, Internat. J. Bifur. Chaos Appl. Sci. Engrg. 2 (1992), 795-813.

4. J. Milnor, On the concept of attractor, Comm. Math. Phys. 99 (1985), 177-195.

5. Ju. S. Il'yashenko, The concept of minimal attractor and maximal attractors of partial differential equations of the Kuramoto-Sivashinsky type, Chaos 1 (1991), 168-173.

6. I. Kan, Intermingled basins, Ergodic Theory Dynamical Systems (to appear).

7. M. Hirsch, C. Pugh, and M. Shub, Invariant manifolds, Lecture Notes in Math., vol. 583, Springer-Verlag, New York, 1977.

8. Y. B. Pesin, Characteristic Lyapunov exponents and smooth ergodic theory, Russian Math. Surveys 32 (1977), 55-114.

9. A. B. Katok and J. M. Strelcyn, Invariant manifolds, entropy and billiards: Smooth maps with singularities, Lecture Notes in Math., vol. 1222, Springer-Verlag, New York, 1986.

10. C. Pugh and M. Shub, Ergodic attractors, Trans. Amer. Math. Soc. 312 (1989), 1-54.

Department of Mathematics, George Mason University, Fairfax, Virginia 22030

E-mail address: ikan@mason 1.gmu.edu 